\title{
Multiblock copolymer synthesis via RAFT emulsion polymerization: Effects of chain mobility within particles on control over molecular weight distribution
}

\author{
Glenn K. K. Clothier, ${ }^{\dagger}$ Thiago R. Guimarães, ${ }^{\dagger}$ Graeme Moad, ${ }^{\dagger}$ Per B. Zetterlund ${ }^{*}{ }^{\dagger}$ \\ $\dagger$ Cluster for Advanced Macromolecular Design (CAMD), School of Chemical Engineering, The University of \\ New South Wales, Sydney, NSW 2052, Australia \\ † CSIRO Manufacturing, Bag 10, Clayton South, VIC 3169, Australia
}

\section{Methylation Procedures}

All samples were methylated before SEC analysis. $50 \mu \mathrm{L}$ of latex and $1 \mathrm{~mL}$ of water were added to a $25 \mathrm{~mL}$ vial, alongside enough $0.1 \mathrm{M} \mathrm{HCl}$ such that the resultant mixture had a $\mathrm{pH}$ of 3-4. $20 \mathrm{~mL}$ of THF was then added with stirring subsequently being conducted via magnetic stir bar. Enough tri(methylsilyl)diazomethane was added such that the solution was tinted with a faint green colour, before being left under stirring for 4 hours. The solutions were checked regularly; if the colour of the solution had become clear, more tri(methylsilyl)diazomethane was added.

Table SI 1 - Conditions and results for the synthesis of PMAA $_{62}-b-\mathrm{PMMA}_{11}$-TTC.

\begin{tabular}{|c|c|}
\hline & $\begin{array}{c}\text { PMAA }_{62}-b- \\
\text { PMMA }_{11^{2}} \text { TTC } \\
\end{array}$ \\
\hline \multicolumn{2}{|l|}{$1^{\text {st }}$ Stage } \\
\hline$[\mathrm{ACPA}]\left(\mathrm{mmol} \mathrm{L}^{-1}\right)$ & 3.01 \\
\hline$[\mathrm{MAA}]\left(\mathrm{mol} \mathrm{L}^{-1}\right)$ & 2.43 \\
\hline$[\mathrm{RAFT}] /[\mathrm{I}]$ & 20 \\
\hline$[\mathrm{MAA}] /[\mathrm{RAFT}]$ & 41 \\
\hline$X_{M A A}(\%) / \mathrm{t}(\mathrm{h})$ & $74 / 5$ \\
\hline$L(\%)$ & 95.4 \\
\hline \multicolumn{2}{|l|}{$2^{\text {nd }}$ Stage } \\
\hline$[\mathrm{MMA}]\left(\mathrm{mmol} \mathrm{L}^{-1}\right)$ & 0.50 \\
\hline [MMA]/[RAFT] & 9.9 \\
\hline$X_{M A A}(\%) / \mathrm{t}(\mathrm{h})$ & $96 / 22$ \\
\hline$X_{M M A}(\%) / \mathrm{t}(\mathrm{h})$ & $89 / 22$ \\
\hline$M_{\mathrm{n}, \text { theo }}$ & 4675 \\
\hline $\boldsymbol{M}_{\mathrm{n}, \mathbf{e x p}} / \bigoplus(\mathrm{THF})$ & $6200 / 1.16$ \\
\hline$M_{\mathrm{n}, \mathrm{exp}} / \bigoplus(\mathrm{DMAc})$ & $6200 / 1.18$ \\
\hline$L(\%)$ & 94.3 \\
\hline
\end{tabular}

$M_{\mathrm{n}}$ values have been recalculated to show non-methylated mass of polymer. 


\section{DP of PMAA P2$_{2}-b-$ PMMA $_{11}$-TTC}

The DP of the hydrophilic PMAA and hydrophobic PMMA blocks were targeted to be 41 and 9.9 units, respectively. The individual conversions for each monomer $\left(\mathrm{X}_{\mathrm{MAA}}=96 \%\right.$ and $\mathrm{X}_{\mathrm{MMA}}=89 \%$, Table S1) when considered beget DPs for PMAA and PMMA of 39.4 and 8.8, respectively. Experimental DP values determined through SEC in DMAc and THF solvents however differed from theoretical calculations, both giving molecular weights of $6000 \mathrm{~g} \mathrm{~mol}^{-}$ 1, higher than predicted $\left(M_{n \text {, theo }}=4675 \mathrm{~g} \mathrm{~mol}^{-1}\right)$; the former value $\left(6000 \mathrm{~g} \mathrm{~mol}^{-1}\right)$ was applied in calculations involving macroRAFT molecular weight. More information regarding this discrepancy can be referred to in the SI of our previous work. ${ }^{1}$

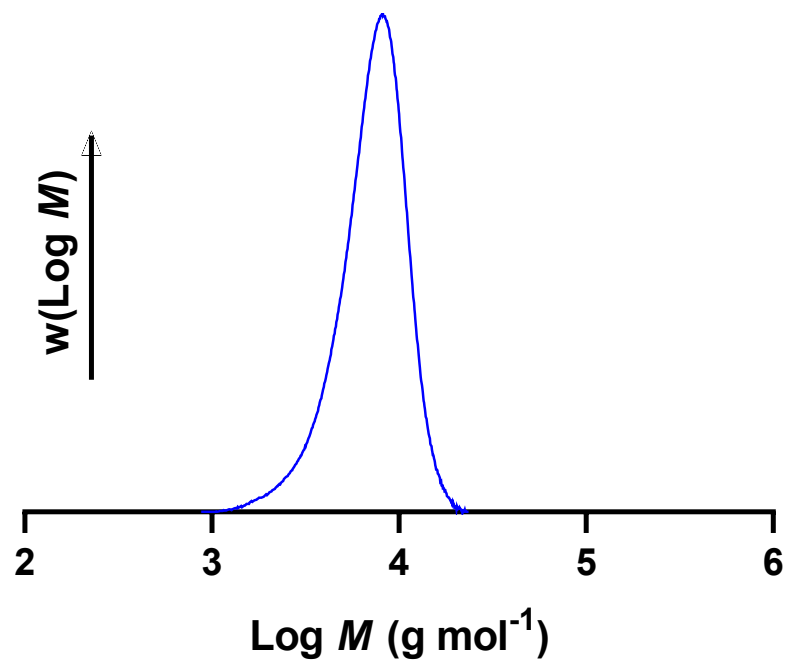

Figure SI 1 SEC chromatogram of PMAA62-b-PMMA11-TTC macroRAFT

\section{Calculating theoretical monomer weight fraction within particles as a function of DP}

For a given DP, monomer (and toluene) weight fraction was calculated as per the following equation, where $\mathrm{m}_{\text {Mon }}$ is mass of monomer and $\mathrm{m}_{\text {Poly }}$ is mass of polymer. The monomer weight fraction was then plotted as a function of DP (Figure $3 \mathrm{a}$ ).

$$
w_{x}=\frac{m_{M o n}}{m_{M o n}+m_{\text {Poly }}}
$$

\section{Calculating theoretical monomer to RAFT concentration ratio within particles as a function of DP}

Monomer concentration was calculated via iterative calculations, keeping in mind that the saturated monomer concentration of $n \mathrm{BMA}$ within polymer particles was capped at $3.8 \mathrm{~mol} \mathrm{~L}^{-}$ 1.2 The first set of calculations aimed at estimating monomer concentration within particles, where $[\mathrm{M}]_{\mathrm{i}=0}$ is the first estimate of monomer concentration inside particles, $\mathrm{n}_{\mathrm{Mon}}$ is the total moles of monomer in the system and $\mathrm{V}_{\text {Poly }}$ is the total polymer volume.

$$
m_{\text {Poly }}=m_{\text {Seed }}+m_{M o n, 0} \times X
$$




$$
\begin{gathered}
\boldsymbol{n}_{\text {Mon }}=\frac{\boldsymbol{m}_{\text {Mon, } 0} \times(1-X)}{M M_{M o n}} \\
V_{\text {Poly }}=\frac{m_{\text {Poly }}}{\rho_{\text {Poly }}} \\
{[M]_{i=0}=\frac{n_{M o n}}{V_{\text {Poly }}}}
\end{gathered}
$$

The subscript $i$ refers to the iteration number. Given the saturated concentration of monomer within particles, $[\mathrm{M}]$ could not exceed $3.8 \mathrm{~mol} \mathrm{~L}^{-1}$; if local monomer concentration did exceed this value it was capped. 3 further iterations were made of the following set of equations, where $\mathrm{V}_{\mathrm{Mon}}$ is the volume of monomer swelling polymer particles, $\mathrm{MM}_{\mathrm{Mon}}$ is the molecular mass of monomer, $\rho_{\text {Mon }}$ is the density of monomer and $V_{\text {Total }}$ is the total particle volume.

$$
\begin{aligned}
n_{M o n, i=1}= & {[M]_{i=0} \times V_{P o l y} } \\
V_{M o n, i=0} & =\frac{n_{M o n, i=1} \times M M_{M o n}}{\rho_{\text {Mon }}} \\
V_{\text {Total }} & =V_{\text {Mon }}+V_{\text {Poly }} \\
{[M]_{i=1} } & =\frac{n_{\text {Mon }, i=1}}{V_{\text {Total }}}
\end{aligned}
$$

The whole process was repeated for every block. The monomer concentration was divided by

\begin{tabular}{|c|c|c|c|c|c|c|c|c|c|c|c|}
\hline Block & $\mathbf{1}^{\mathrm{a}}$ & 2 & 3 & 4 & 5 & 6 & 7 & 8 & 9 & 10 & 11 \\
\hline DP $_{\text {nBMA }}$ & 200 & 100 & 100 & 100 & 100 & 100 & 100 & 100 & 100 & 100 & 100 \\
\hline $\operatorname{MacroRAFT}(\mathrm{g})^{\mathrm{b}}$ & 0.89 & - & - & - & - & - & - & - & - & - & - \\
\hline Water $(\mathrm{g})$ & 24.7 & 15.8 & 11.4 & 11.0 & 10.8 & 10.4 & 10.2 & 10.1 & 9.9 & 9.7 & 9.5 \\
\hline$n$ BMA (g) & 4.2 & 2.09 & 2.02 & 1.95 & 1.89 & 1.84 & 1.80 & 1.77 & 1.73 & 1.70 & 1.68 \\
\hline $\mathrm{NaOH}(\mathrm{g})$ & 0.25 & - & - & - & - & - & - & - & - & - & - \\
\hline KPS (mg) & 1.42 & 1.91 & - & - & - & - & - & - & - & - & - \\
\hline Sample (g) & 0 & -2 & -2 & -2 & -2 & -2 & -2 & -2 & -2 & -2 & -2 \\
\hline Time (min) & 120 & 45 & 30 & 30 & 30 & 30 & 30 & 30 & 30 & 30 & 30 \\
\hline $\mathrm{SC}_{\mathrm{th}}(\%)^{\mathrm{c}}$ & 17.0 & 14.7 & 14.8 & 14.8 & 14.8 & 14.9 & 14.9 & 14.9 & 14.9 & 14.9 & 14.9 \\
\hline$[\mathrm{RAFT}] /[\mathrm{I}]$ & 28.2 & 15 & - & - & - & - & - & - & - & - & - \\
\hline $\mathrm{M}_{\mathrm{n}, \text { theo }}\left(\mathrm{g} \mathrm{mol}^{-1}\right)^{\mathrm{d}}$ & 34,400 & 48,700 & 63,000 & 77,400 & 91,700 & 106,100 & 120,500 & 135,000 & 149,500 & 163,900 & 178,400 \\
\hline $\mathrm{M}_{\mathrm{n}, \exp }\left(\mathrm{g} \mathrm{mol}^{-1}\right)^{\mathrm{e}}$ & 24,700 & 29,300 & 34,200 & 39,800 & 46,100 & 53,000 & 56,800 & 64,800 & 70,200 & 74,100 & 77,100 \\
\hline $\mathrm{D}^{\mathrm{e}}$ & 1.09 & 1.09 & 1.10 & 1.13 & 1.16 & 1.16 & 1.21 & 1.19 & 1.34 & 1.27 & 1.38 \\
\hline$X(\%)^{f}$ & 99.9 & 97.2 & 100.0 & 99.8 & 100.0 & 100.0 & 100.0 & 99.7 & 100.0 & 100.0 & 98.7 \\
\hline
\end{tabular}
RAFT concentration and plotted as a function of DP (Figure $3 \mathrm{~b}$ ). The principle of this iterative process is that the monomer concentration calculated by eqn SI 6 is sufficiently close to the value of the monomer concentration used as input value in eqn SI 3 - the iterative process is repeated until this is the case (within $0.1 \%$; typically less than 5 iterations).

Table SI 2 - Experimental conditions for the synthesis of P $n$ BMA undecablock homopolymer (Exp 1) via PMAA $_{62}-b$-PMMA $11-T T C$ assisted emulsion polymerization. 


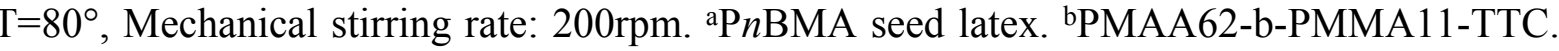
${ }^{\mathrm{c}}$ Theoretical solids content. ${ }^{\mathrm{d}}$ Theoretical number-average molar mass determined via Equation 2. Experimental number-average molar mass and dispersity determined via DMAC-SEC using PMMA calibration. ${ }^{f}$ Overall conversion determined via ${ }^{1} \mathrm{H}$ NMR. ${ }^{\mathrm{g}}$ Degree of livingness calculated using Equation 1.

Table SI 3 - Experimental conditions for the synthesis of P $n$ BMA triblock homopolymer (Exp 2) via $\mathrm{PMAA}_{62}-b$-PMMA $11-\mathrm{TTC}$ assisted emulsion polymerization.

\begin{tabular}{|c|c|c|c|}
\hline Block & $\mathbf{1}^{\mathrm{a}}$ & 2 & 3 \\
\hline DP $_{\text {nBMA }}$ & 200 & 500 & 500 \\
\hline $\operatorname{MacroRAFT}(\mathrm{g})^{\mathrm{b}}$ & 0.89 & - & - \\
\hline Water (g) & 24.7 & 63.6 & 58.3 \\
\hline$n$ BMA (g) & 4.20 & 10.52 & 10.31 \\
\hline $\mathrm{NaOH}(\mathrm{g})$ & 0.25 & - & - \\
\hline KPS (mg) & 1.42 & 2.00 & - \\
\hline Sample (g) & 0 & -2 & -2 \\
\hline Time (min) & 120 & 150 & 150 \\
\hline $\mathrm{SC}_{\text {th }}(\%)^{\mathrm{c}}$ & 17.0 & 15.0 & 15.0 \\
\hline$[\mathrm{RAFT}] /[\mathrm{I}]$ & 28.2 & 14.6 & - \\
\hline $\mathrm{M}_{\mathrm{n}, \text { theo }}\left(\mathrm{g} \mathrm{mol}^{-1}\right)^{\mathrm{d}}$ & 34,400 & 105,600 & 176,700 \\
\hline $\mathrm{M}_{\mathrm{n}, \exp }\left(\mathrm{g} \mathrm{mol}^{-1}\right)^{\mathrm{e}}$ & 24,700 & 62,600 & 85,600 \\
\hline $\mathrm{D}^{\mathrm{e}}$ & 1.09 & 1.20 & 1.33 \\
\hline$X(\%)^{f}$ & 99.9 & 100.0 & 98.9 \\
\hline $\mathrm{L}(\%)^{\mathrm{g}}$ & 93.7 & 92.3 & 90.1 \\
\hline
\end{tabular}

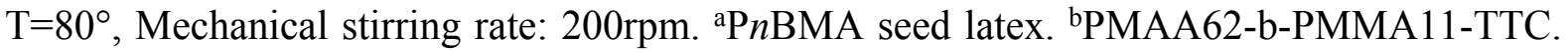
${ }^{\mathrm{c}}$ Theoretical solids content. ${ }^{\mathrm{d}}$ Theoretical number-average molar mass determined via Equation 2. 'Experimental number-average molar mass and dispersity determined via DMAC-SEC using PMMA calibration. ${ }^{\mathrm{f}}$ Overall conversion determined via ${ }^{1} \mathrm{H}$ NMR. ${ }^{\mathrm{g}}$ Degree of livingness calculated using Equation 1.

Table SI 4 - Experimental conditions for the synthesis of P $t$ BMA undecablock homopolymer (Exp 3) via PMAA $_{62}-b$-PMMA $11-$ TTC assisted emulsion.

\begin{tabular}{|c|c|c|c|c|c|c|c|c|c|c|c|}
\hline Block & $\mathbf{1}^{\mathbf{a}}$ & $\mathbf{2}$ & $\mathbf{3}$ & $\mathbf{4}$ & $\mathbf{5}$ & $\mathbf{6}$ & $\mathbf{7}$ & $\mathbf{8}$ & $\mathbf{9}$ & $\mathbf{1 0}$ & $\mathbf{1 1}$ \\
\hline DP $_{\text {nBMA }}$ & $\mathbf{2 0 0}$ & $\mathbf{1 0 0}$ & $\mathbf{1 0 0}$ & $\mathbf{1 0 0}$ & $\mathbf{1 0 0}$ & $\mathbf{1 0 0}$ & $\mathbf{1 0 0}$ & $\mathbf{1 0 0}$ & $\mathbf{1 0 0}$ & $\mathbf{1 0 0}$ & $\mathbf{1 0 0}$ \\
\hline MacroRAFT (g) & 0.89 & - & - & - & - & - & - & - & - & - & - \\
\hline Water (g) & 24.7 & 15.8 & 11.4 & 11.0 & 10.7 & 10.4 & 10.2 & 10.0 & 9.9 & 9.7 & 9.5 \\
\hline$n$ BMA (g) & 4.20 & 2.11 & 2.02 & 1.95 & 1.89 & 1.85 & 1.81 & 1.77 & 1.74 & 1.71 & 1.68 \\
\hline $\mathrm{NaOH} \mathrm{(g)}$ & 0.25 & - & - & - & - & - & - & - & - & - & - \\
\hline $\mathrm{KPS}(\mathrm{mg})$ & 1.42 & 2.00 & - & - & - & - & - & - & - & - & - \\
\hline Sample (g) & 0 & -2 & -2 & -2 & -2 & -2 & -2 & -2 & -2 & -2 & -2 \\
\hline Time (min) & 120 & 45 & 30 & 30 & 30 & 30 & 30 & 30 & 30 & 30 & 30 \\
\hline $\mathrm{SC}_{\text {th }}(\%)^{\mathrm{c}}$ & 17.0 & 15.0 & 15.0 & 15.0 & 15.0 & 15.0 & 15.0 & 15.0 & 15.0 & 15.0 & 15.0 \\
\hline$\left[\mathrm{RAFT}_{/ \mathrm{I}} \mathrm{I}\right]$ & 28.2 & 14.7 & - & - & - & - & - & - & - & - & - \\
\hline
\end{tabular}




\begin{tabular}{|c|c|c|c|c|c|c|c|c|c|c|c|}
\hline $\mathrm{M}_{\mathrm{n}, \text { theo }}\left(\mathrm{g} \mathrm{mol}^{-1}\right)^{\mathrm{d}}$ & 34,400 & 48,700 & 63,000 & 77,400 & 91,700 & 106,100 & 120,500 & 135,000 & 149,500 & 163,900 & 178,400 \\
\hline $\mathrm{M}_{\mathrm{n}, \exp }\left(\mathrm{g} \mathrm{mol}^{-1}\right)^{\mathrm{e}}$ & 24,700 & 28,500 & 36,900 & 43,200 & 48,700 & 54,300 & 57,300 & 58,800 & 64,000 & 68,000 & 71,600 \\
\hline $\mathrm{D}^{\mathrm{e}}$ & 1.09 & 1.07 & 1.20 & 1.48 & 1.88 & 2.26 & 2.66 & 2.92 & 3.21 & 3.36 & 3.51 \\
\hline $\mathrm{X} \mathrm{( \% )^{ \textrm {f } }}$ & 99.9 & 98.9 & 99.3 & 99.1 & 99.1 & 98.6 & 98.8 & 98.7 & 98.5 & 98.5 & 97.7 \\
\hline $\mathrm{L}(\%)^{\mathrm{g}}$ & 93.7 & 93.1 & 92.7 & 92.3 & 92.0 & 91.6 & 91.2 & 90.8 & 90.4 & 90.0 & 89.7 \\
\hline
\end{tabular}

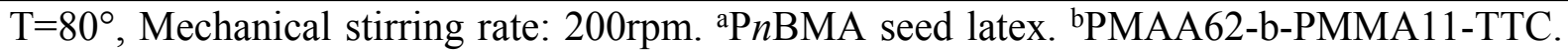
${ }^{\mathrm{c}}$ Theoretical solids content. ${ }^{\mathrm{d}}$ Theoretical number-average molar mass determined via Equation

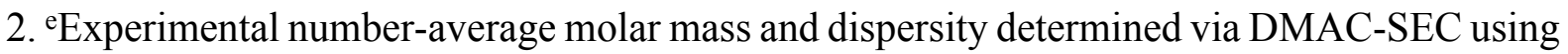

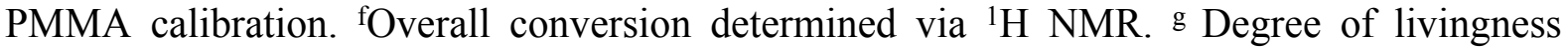
calculated using Equation 1.

Table SI 5 - Experimental conditions for the synthesis of P $t$ BMA triblock homopolymer (Exp 4) via $\mathrm{PMAA}_{62}-b-\mathrm{PMMA}_{11}-\mathrm{TTC}$ assisted emulsion polymerization.

\begin{tabular}{|c|c|c|c|}
\hline Block & $\mathbf{1}^{\mathrm{a}}$ & 2 & 3 \\
\hline $\mathbf{D P}_{\text {nBMA }}$ & 200 & 500 & 500 \\
\hline MacroRAFT $(\mathrm{g})^{\mathrm{b}}$ & 0.89 & - & - \\
\hline Water $(\mathrm{g})$ & 24.7 & 63.5 & 58.2 \\
\hline$n$ BMA (g) & 4.20 & 10.52 & 10.30 \\
\hline $\mathrm{NaOH}(\mathrm{g})$ & 0.25 & - & - \\
\hline KPS (mg) & 1.42 & 2.00 & - \\
\hline Sample (g) & 0 & -2 & -2 \\
\hline Time (min) & 120 & 150 & 150 \\
\hline $\mathrm{SC}_{\text {th }}(\%)^{\mathrm{c}}$ & 17.0 & 15.0 & 15.0 \\
\hline$[\mathrm{RAFT}] /[\mathrm{I}]$ & 28.2 & 14.5 & - \\
\hline $\mathrm{M}_{\mathrm{n}, \text { theo }}\left(\mathrm{g} \mathrm{mol}^{-1}\right)^{\mathrm{d}}$ & 34,400 & 105,600 & 176,700 \\
\hline $\mathrm{M}_{\mathrm{n}, \exp }\left(\mathrm{g} \mathrm{mol}^{-1}\right)^{\mathrm{e}}$ & 24,700 & 62,600 & 85,600 \\
\hline $\mathrm{Ð}^{\mathrm{e}}$ & 1.09 & 1.36 & 2.04 \\
\hline$X(\%)^{f}$ & 99.9 & 98.8 & 99.1 \\
\hline $\mathrm{L}(\%)^{\mathrm{g}}$ & 93.7 & 92.3 & 90.1 \\
\hline
\end{tabular}

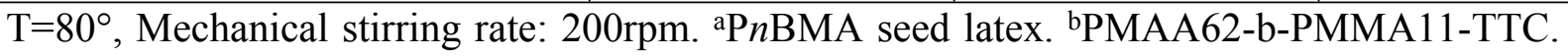
${ }^{\mathrm{c}}$ Theoretical solids content. ${ }^{\mathrm{d}}$ Theoretical number-average molar mass determined via Equation 2. 'Experimental number-average molar mass and dispersity determined via DMAC-SEC using PMMA calibration. fOverall conversion determined via ${ }^{1} \mathrm{H}$ NMR. ${ }^{\mathrm{g}}$ Degree of livingness calculated using Equation 1.

Table SI 6 - Experimental conditions for the synthesis of PiBMA undecablock homopolymer (Exp 5) via $\mathrm{PMAA}_{62}-b$-PMMA $11-\mathrm{TTC}$ assisted emulsion polymerization.

\begin{tabular}{|c|c|c|c|c|c|c|c|c|c|c|c|}
\hline Block & $\mathbf{1}^{\mathbf{a}}$ & $\mathbf{2}$ & $\mathbf{3}$ & $\mathbf{4}$ & $\mathbf{5}$ & $\mathbf{6}$ & $\mathbf{7}$ & $\mathbf{8}$ & $\mathbf{9}$ & $\mathbf{1 0}$ & $\mathbf{1 1}$ \\
\hline DP $_{\text {nBMA }}$ & $\mathbf{2 0 0}$ & $\mathbf{1 0 0}$ & $\mathbf{1 0 0}$ & $\mathbf{1 0 0}$ & $\mathbf{1 0 0}$ & $\mathbf{1 0 0}$ & $\mathbf{1 0 0}$ & $\mathbf{1 0 0}$ & $\mathbf{1 0 0}$ & $\mathbf{1 0 0}$ & $\mathbf{1 0 0}$ \\
\hline MacroRAFT (g) & 0.88 & - & - & - & - & - & - & - & - & - & - \\
\hline Water $(\mathrm{g})$ & 24.7 & 15.9 & 11.4 & 11.0 & 10.8 & 10.4 & 10.2 & 10.0 & 9.8 & 9.7 & 9.5 \\
\hline$n$ BMA (g) & 4.20 & 2.10 & 2.01 & 1.95 & 1.89 & 1.84 & 1.80 & 1.77 & 1.74 & 1.70 & 1.68 \\
\hline NaOH (g) & 0.24 & - & - & - & - & - & - & - & - & - & - \\
\hline KPS (mg) & 1.39 & 2.00 & - & - & - & - & - & - & - & - & - \\
\hline
\end{tabular}




\begin{tabular}{|c|c|c|c|c|c|c|c|c|c|c|c|}
\hline Sample (g) & 0 & -2 & -2 & -2 & -2 & -2 & -2 & -2 & -2 & -2 & -2 \\
\hline Time $(\mathrm{min})$ & 120 & 45 & 30 & 30 & 30 & 30 & 30 & 30 & 30 & 30 & 30 \\
\hline $\mathrm{SC}_{\text {th }}(\%)^{\mathrm{c}}$ & 17.0 & 14.9 & 15.0 & 15.0 & 15.0 & 15.0 & 15.0 & 15.0 & 15.0 & 15.0 & 15.0 \\
\hline$[\mathrm{RAFT}] /[\mathrm{I}]$ & 28.6 & 14.6 & - & - & - & - & - & - & - & - & - \\
\hline $\mathrm{M}_{\mathrm{n}, \text { theo }}\left(\mathrm{g} \mathrm{mol}^{-1} \mathrm{~d}^{\mathrm{d}}\right.$ & 34,400 & 48,700 & 63,000 & 77,400 & 91,700 & 106,100 & 120,500 & 135,000 & 149,500 & 163,900 & 178,400 \\
\hline $\mathrm{M}_{\mathrm{n}, \text { exp }}\left(\mathrm{g} \mathrm{mol}^{-1}\right)^{\mathrm{e}}$ & 23,100 & 32,700 & 39,300 & 45,400 & 52,500 & 58,600 & 65,500 & 70,100 & 75,400 & 77,400 & 81,900 \\
\hline $\mathrm{Ð}^{\mathrm{e}}$ & 1.13 & 1.08 & 1.11 & 1.16 & 1.21 & 1.30 & 1.36 & 1.45 & 1.51 & 1.65 & 1.69 \\
\hline $\mathrm{X} \mathrm{( \% )^{ \textrm {f } }}$ & 99.9 & 98.9 & 99.3 & 99.1 & 99.1 & 98.6 & 98.8 & 98.7 & 98.5 & 98.5 & 97.7 \\
\hline $\mathrm{L}(\%)^{\mathrm{g}}$ & 93.7 & 93.1 & 92.7 & 92.3 & 92.0 & 91.6 & 91.2 & 90.8 & 90.4 & 90.0 & 89.6 \\
\hline
\end{tabular}

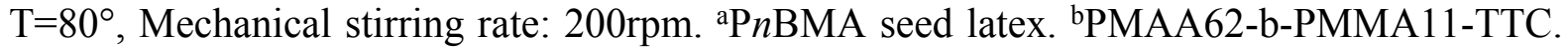

${ }^{\mathrm{c}}$ Theoretical solids content. ${ }^{\mathrm{d}}$ Theoretical number-average molar mass determined via Equation

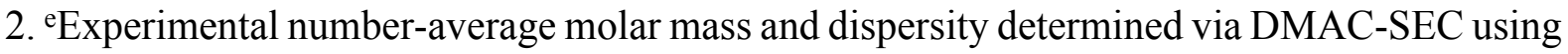
PMMA calibration. fOverall conversion determined via ${ }^{1} \mathrm{H}$ NMR. ${ }^{\mathrm{g}}$ Degree of livingness calculated using Equation 1.

Table SI 7 - Experimental conditions for the synthesis of P $n$ BMA decablock homopolymer (Exp 6) via PMAA $_{62}-b$-PMMA $11-$ TTC assisted emulsion polymerization.

\begin{tabular}{|c|c|c|c|c|c|c|c|c|c|c|}
\hline Block & $\mathbf{1}^{\mathrm{a}}$ & 2 & 3 & 4 & 5 & 6 & 7 & 8 & 9 & 10 \\
\hline DP $_{\text {nBMA }}$ & 275 & 100 & 100 & 100 & 100 & 100 & 100 & 100 & 100 & 100 \\
\hline MacroRAFT (g) & 0.87 & - & - & - & - & - & - & - & - & - \\
\hline Water $(\mathrm{g})$ & 23.5 & 23.3 & - & 1.7 & 7.3 & 6.9 & 6.6 & 6.4 & 6.4 & 6.2 \\
\hline$n$ BMA (g) & 5.40 & 1.92 & 2.12 & 2.07 & 1.77 & 1.71 & 1.65 & 1.62 & 1.58 & 1.54 \\
\hline $\mathrm{NaOH}(\mathrm{g})$ & 0.23 & - & - & - & - & - & - & - & - & - \\
\hline KPS (mg) & 1.40 & 2.00 & - & - & - & - & - & - & - & - \\
\hline Sample (g) & 0 & -2 & -2 & -2 & -2 & -2 & -2 & -2 & -2 & -2 \\
\hline Time (min) & 120 & 45 & 35 & 35 & 35 & 35 & 35 & 35 & 35 & 35 \\
\hline $\mathrm{SC}_{\mathrm{th}}(\%)^{\mathrm{c}}$ & 20.9 & 14.9 & 18.3 & 21.0 & 20.2 & 21.0 & 21.1 & 21.2 & 21.3 & 21.4 \\
\hline$[\mathrm{RAFT}] /[\mathrm{I}]$ & 29.0 & 13.3 & - & - & - & - & - & - & - & - \\
\hline $\mathrm{M}_{\mathrm{n}, \text { theo }}\left(\mathrm{g} \mathrm{mol}^{-1}\right)^{\mathrm{d}}$ & 45,300 & 59,500 & 73,700 & 88,000 & 102,300 & 116,500 & 130,700 & 145,000 & 159,200 & 173,400 \\
\hline $\mathrm{M}_{\mathrm{n}, \exp }\left(\mathrm{g} \mathrm{mol}^{-1}\right)^{\mathrm{e}}$ & 35,000 & 39,000 & 47,200 & 57,500 & 63,100 & 73,400 & 87,400 & 93,000 & 98,000 & 104,800 \\
\hline$\bigoplus^{\mathrm{e}}$ & 1.09 & 1.12 & 1.17 & 1.22 & 1.26 & 1.31 & 1.36 & 1.47 & 1.556 & 1.64 \\
\hline$X(\%)^{f}$ & 99.9 & 99.0 & 98.7 & 98.9 & 98.8 & 98.6 & 98.7 & 98.2 & 97.1 & 97.0 \\
\hline $\mathrm{L}(\%)^{\mathrm{g}}$ & 93.7 & 93.1 & 92.6 & 92.1 & 91.6 & 91.1 & 90.6 & 90.2 & 89.7 & 89.2 \\
\hline
\end{tabular}

$\mathrm{T}=80^{\circ}$, Mechanical stirring rate: $200 \mathrm{rpm} .{ }^{\text {aP }} n$ BMA seed latex. ${ }^{\text {bPMAA62-b-PMMA11-TTC. }}$ ${ }^{\mathrm{c}}$ Theoretical solids content. ${ }^{\mathrm{d}}$ Theoretical number-average molar mass determined via Equation 2. Experimental number-average molar mass and dispersity determined via DMAC-SEC using PMMA calibration. ${ }^{f}$ Overall conversion determined via ${ }^{1} \mathrm{H}$ NMR. ${ }^{\mathrm{g}}$ Degree of livingness calculated using Equation 1. 
Table SI 8 - Experimental conditions for the synthesis of PiBMA decablock homopolymer (Exp 7) via PMAA $_{62}-b$ - $\mathrm{PMMA}_{11}-\mathrm{TTC}$ assisted emulsion polymerization.

\begin{tabular}{|c|c|c|c|c|c|c|c|c|c|c|}
\hline Block & $\mathbf{1}^{\mathrm{a}}$ & 2 & 3 & 4 & 5 & 6 & 7 & 8 & 9 & 10 \\
\hline DP $_{\text {nBMA }}$ & 200 & 100 & 100 & 100 & 100 & 100 & 100 & 100 & 100 & 100 \\
\hline MacroRAFT $(\mathrm{g})^{\mathrm{b}}$ & 0.89 & - & - & - & - & - & - & - & - & - \\
\hline Water $(\mathrm{g})$ & 24.8 & 15.9 & 11.4 & 11.0 & 10.8 & 10.4 & 10.2 & 10.0 & 9.8 & 9.7 \\
\hline$n$ BMA (g) & 4.22 & 2.10 & 2.01 & 1.95 & 1.89 & 1.84 & 1.80 & 1.77 & 1.74 & 1.70 \\
\hline $\mathrm{NaOH}(\mathrm{g})$ & 0.25 & - & - & - & - & - & - & - & - & - \\
\hline KPS (mg) & 1.40 & 2.00 & - & - & - & - & - & - & - & - \\
\hline Sample (g) & 0 & -2 & -2 & -2 & -2 & -2 & -2 & -2 & -2 & -2 \\
\hline Time (min) & 120 & 45 & 30 & 30 & 30 & 30 & 30 & 30 & 30 & 30 \\
\hline $\mathrm{SC}_{\text {th }}(\%)^{\mathrm{c}}$ & 17.0 & 14.8 & 15.0 & 15.0 & 15.0 & 15.0 & 15.0 & 15.0 & 15.0 & 15.0 \\
\hline$[\mathrm{RAFT}] /[\mathrm{I}]$ & 28.7 & 14.8 & - & - & - & - & - & - & - & - \\
\hline $\mathrm{M}_{\mathrm{n}, \text { theo }}\left(\mathrm{g} \mathrm{mol}^{-1}\right)^{\mathrm{d}}$ & 34,400 & 48,700 & 63,000 & 77,400 & 91,700 & 106,100 & 120,500 & 135,000 & 149,500 & 163,900 \\
\hline $\mathrm{M}_{\mathrm{n}, \exp }\left(\mathrm{g} \mathrm{mol}^{-1}\right)^{\mathrm{e}}$ & 27,400 & 31,100 & 38,600 & 47,700 & 56,700 & 61,700 & 72,800 & 84,900 & 86,300 & 96,000 \\
\hline $\mathrm{D}^{\mathrm{e}}$ & 1.09 & 1.08 & 1.11 & 1.14 & 1.21 & 1.35 & 1.39 & 1.39 & 1.56 & 1.59 \\
\hline$X(\%)^{f}$ & 99.9 & 99.1 & 98.9 & 98.2 & 97.7 & 97.9 & 97.7 & 97.1 & 96.6 & 96.5 \\
\hline $\mathrm{L}(\%)^{\mathrm{g}}$ & 93.7 & 93.1 & 92.7 & 92.4 & 92.0 & 91.6 & 91.2 & 90.8 & 90.4 & 90.1 \\
\hline
\end{tabular}

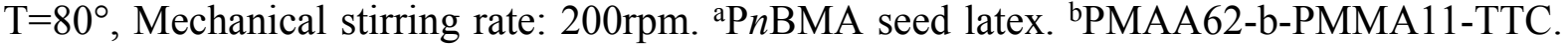
${ }^{\mathrm{c}}$ Theoretical solids content. ${ }^{\mathrm{d}}$ Theoretical number-average molar mass determined via Equation 2. experimental number-average molar mass and dispersity determined via DMAC-SEC using

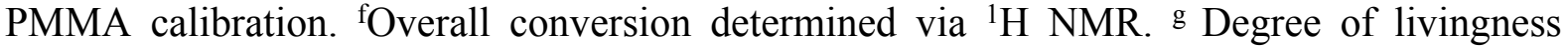
calculated using Equation 1.
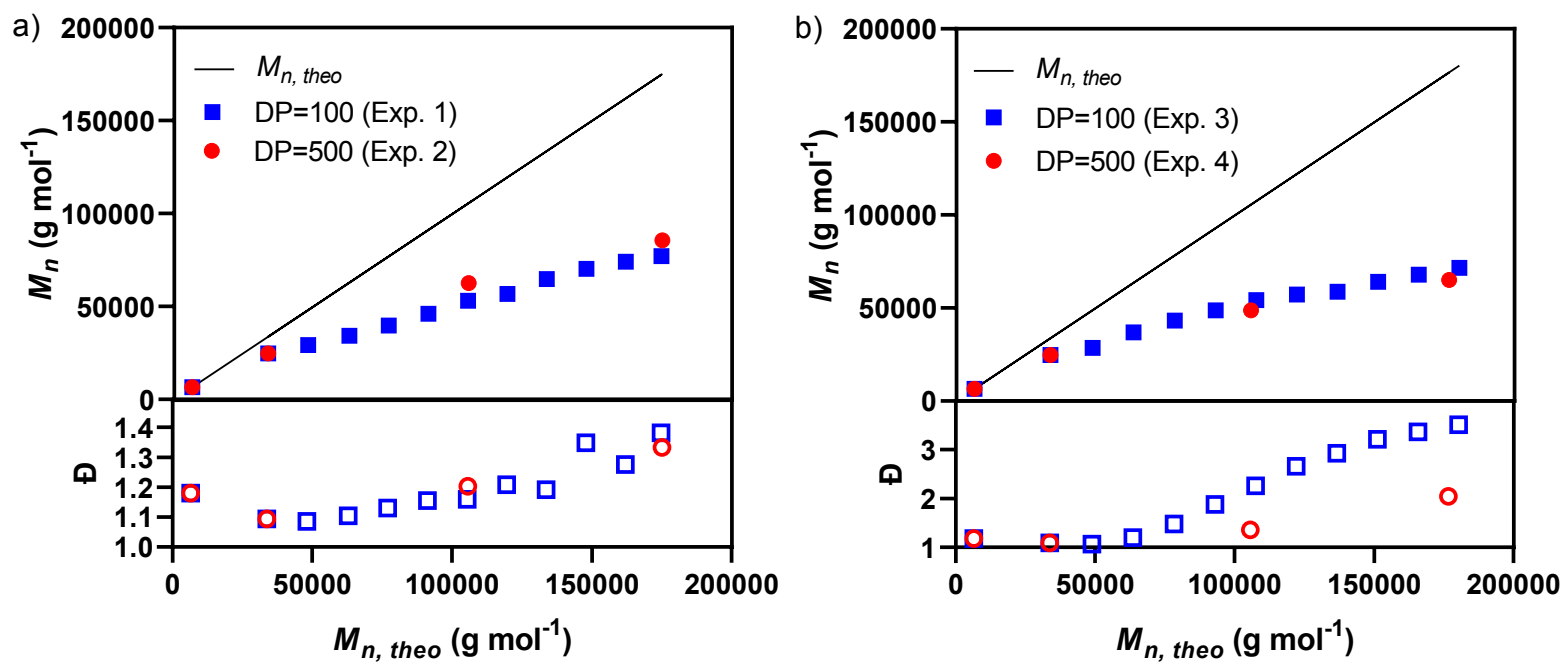
Figure SI 2 Experimental and theoretical molecular weight and dispersity comparisons via DMACSEC for different block DPs of a) PnBMA and b) P $t$ BMA systems.
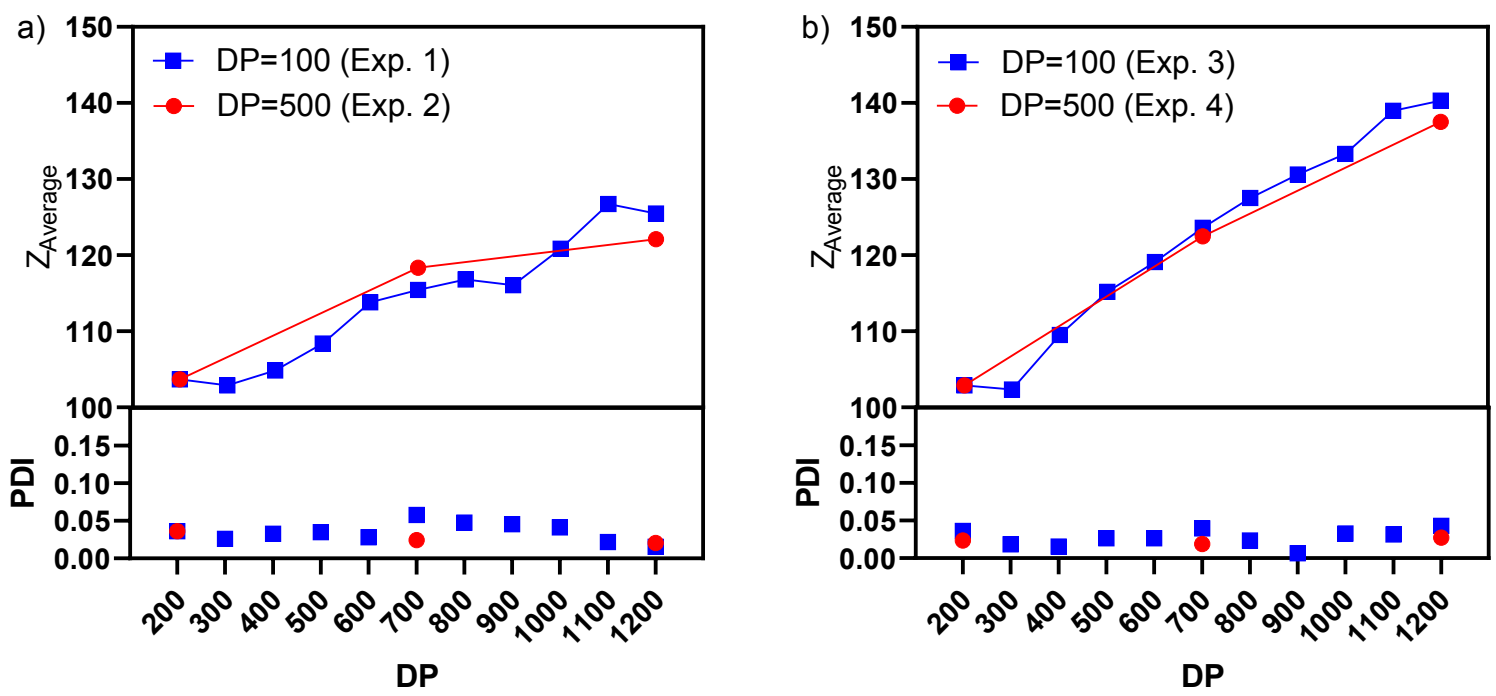

Figure SI 3 Comparison of evolution of particle size and PDI via DLS of a) P $n$ BMA and b) P $t$ BMA systems as a function of DP.

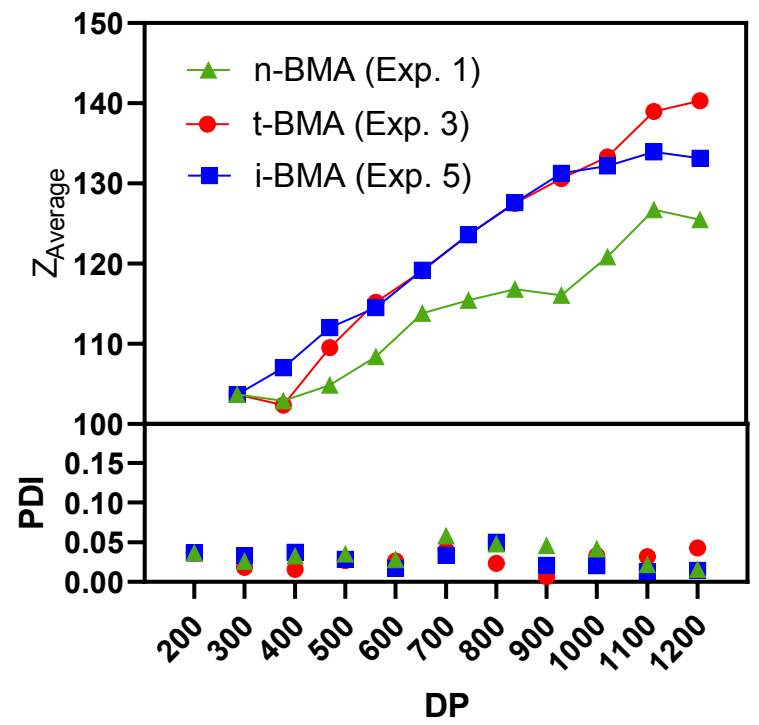

Figure SI 4 Comparison of evolution of particle size and PDI via DLS for $n \mathrm{BMA}, t \mathrm{BMA}$ and $i \mathrm{BMA}$ undecablock systems. 


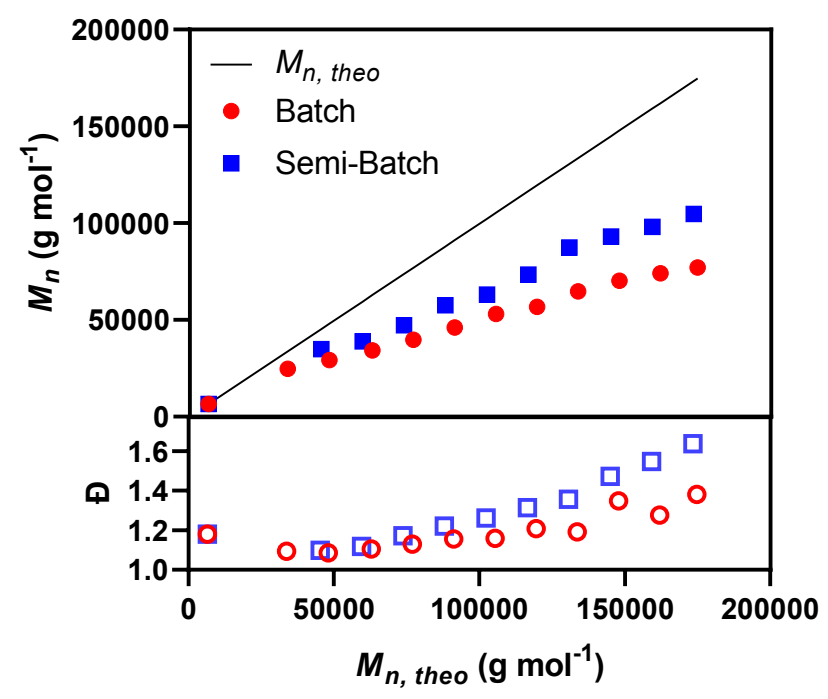

Figure SI 5 Experimental and theoretical molecular weight and dispersity comparisons via DMACSEC for polymerisations of $n$ BMA using different techniques i.e. batch (Exp. 1) and semi-batch (Exp. $6)$.

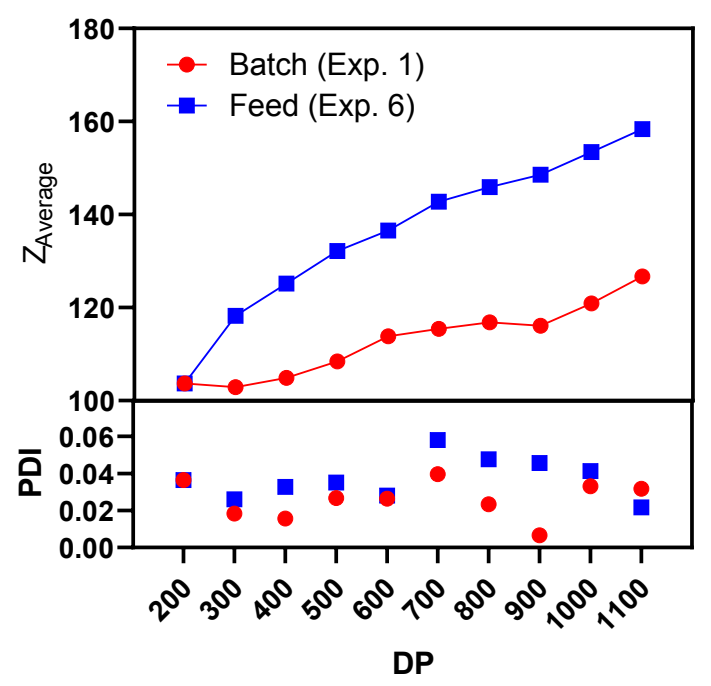

Figure SI 6 Comparison of evolution of particle size and PDI via DLS for polymerisations of $n$ BMA using different techniques i.e. batch (Exp. 1) and semi-batch (Exp. 6). 


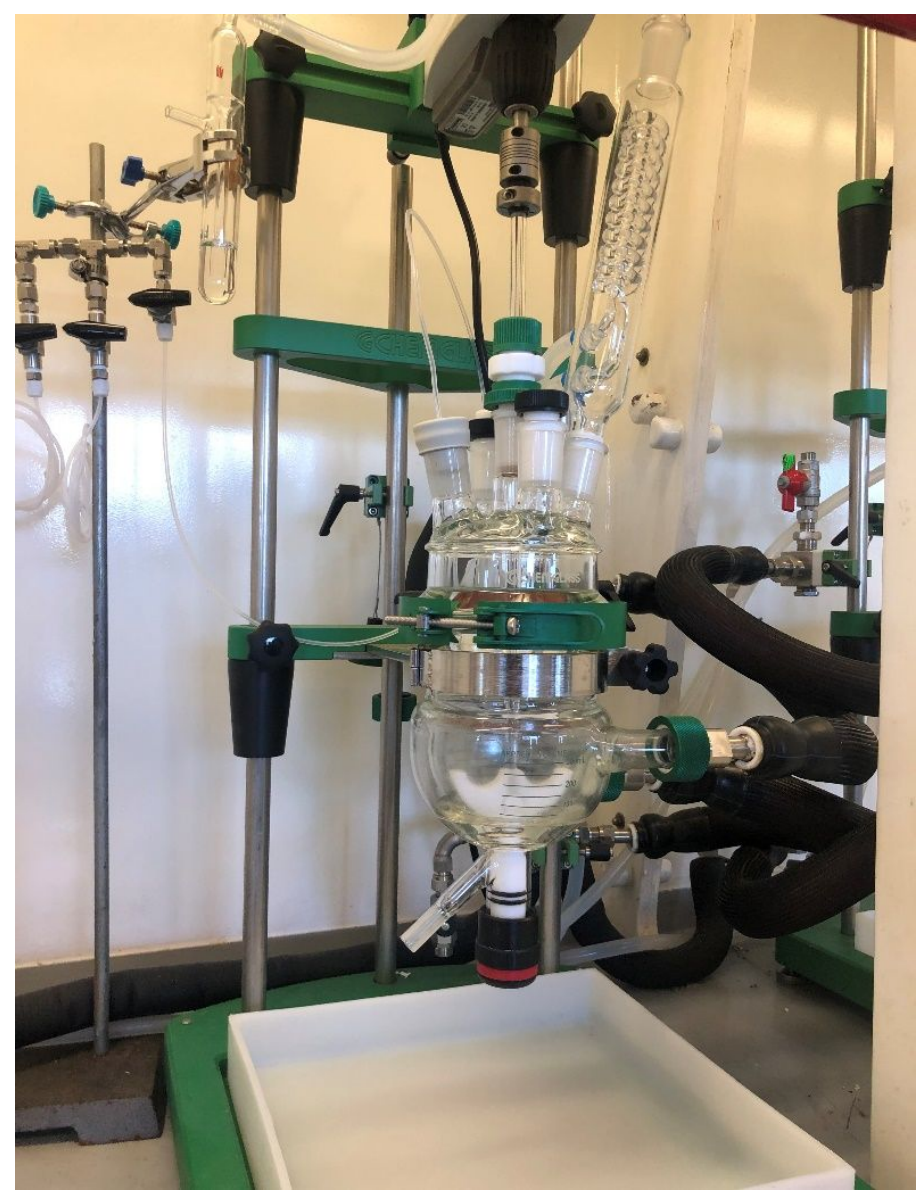

Figure SI $7-300 \mathrm{~mL}$ double jacked reactor with, condenser, $\mathrm{N}_{2}$ inlet tubing and mechanical stirring.

\section{References}

1. Clothier, G. K.; Guimarães, T. R.; Khan, M.; Moad, G.; Perrier, S. b.; Zetterlund, P. B. Exploitation of the nanoreactor concept for efficient synthesis of multiblock copolymers via macroRAFT-mediated emulsion polymerization. ACS Macro Letters 2019, 8 (8), 989-995.

2. Halnan, L. F.; Napper, D. H.; Gilbert, R. G. A study of the kinetics of the emulsion polymerization of butyl methacrylate. Journal of the Chemical Society, Faraday Transactions 1: Physical Chemistry in Condensed Phases 1984, 80 (10), 2851-2865 DOI: 10.1039/F19848002851. 